\title{
Fortalecimiento de asociaciones campesinas del municipio del Tambo mediante la transformación de cultivos tradicionales hacia frutos de alto valor agregado
}

\section{Caso de estudio: Producto Sacha Inchi de la Cooperativa "Sachatambo" del Municipio del Tambo, Cauca, Colombia}

\author{
Diana Ximena Sánchez Tróchez" \\ Institución Universitaria Colegio Mayor del Canca \\ dxsanchez@unimayor.edu.co \\ Orcid: https://orcid.org/0000-0003-0309-0673 \\ Adriana Diago Ortiz \\ Institución Universitaria Colegio Mayor del Canca \\ adiago@unimayor.edu.co \\ Orcid: http://orcid.org/0000-0003-1150-4792 \\ Wilfred Fabián Rivera Martínez \\ Corporación Autónoma del Cauca \\ wilfred.rivera.m@uniautonoma.edu.co \\ Orcid: https://orcid.org/0000-0003-2888-7929 \\ Laura Beatriz Potes Ordoñez \\ Fundación Universitaria de Popayán \\ laura.potes@docente.fup.edu.co \\ Orcid: https://orcid.org/0000-0002-3654-4726 \\ Marly Viviana Navia Mera \\ Institución Universitaria Colegio Mayor del Cauca \\ marly_navia@unimayor.edu.co
}

Recibido: 29/09/2020; Aceptado: 05/11/2020; Publicado: 13/09/2021

"Autor de correspondencia:
dxsanchez@unimayor.edu.co
ISSN 0212-1867 / e-ISSN 1989-3574
@ ESIC Editorial,
ESIC Business \& Marketing School
https://revistasinvestigacion.esic.edu/esicmarket

\begin{abstract}
Cómo citar este artículo
Sánchez Tróchez, D. X.; Diago Ortiz, A.; Rivera Martínez, W. F., Potes Ordoñez, L. B. y Navia Mera, M. V., 2021: Fortalecimiento de asociaciones campesinas del municipio del Tambo mediante la transformación de cultivos tradicionales hacia frutos de alto valor agregado, Esic Market Economics and Business Journal, 52(3), e5235. DOI: 10.7200/esicm.168.
\end{abstract} 0523.5 


\section{Resumen}

Objetivo: Esta investigación se desarrolla bajo la pregunta orientadora: ¿Cuáles son los elementos necesarios para la transformación de cultivos tradicionales hacia productos de alto valor agregado?; desde la perspectiva teórica se asumen los postulados de la modernización de la agricultura de Schultz (1967) y gestión organizacional de Chiavenato (2017).

Metodología: Se basó en investigación descriptiva, bajo los criterios de un estudio de caso con encuestas semiestructuradas que permitieran conocer las situaciones, costumbres y actitudes predominantes a través de la descripción de las actividades, procesos y personas del entorno de la Cooperativa, así como su mercado potencial.

Resultados: La investigación propone cinco elementos basados en economía agraria que permiten a las comunidades, la transformación de cultivos tradicionales hacia productos de alto valor agregado, incrementando su competitividad, y concluye que las organizaciones requieren estrategias que resalten atributos de producto, y mayores esfuerzos en orientación estratégica.

Limitaciones: Los hallazgos de la investigación son extrapolables a organizaciones con características, tamaños y focos similares, entre ellas, empresas campesinas y cooperativas colombianas interesadas en fortalecer su economía agraria. En su lugar, este análisis sirve para conocer con más profundidad el fenómeno en cuestión, pero con posibilidad de réplica en organizaciones interesadas en la transformación del sector rural. Sin embargo, no abarca tejidos empresariales de sectores industrializados.

Implicaciones prácticas: El estudio es de utilidad en áreas de direccionamiento estratégico y gestión de recursos humanos permitiendo visualizar mecanismos de operativización para planes de innovación de producto.

Palabras claves: Economía rural; innovación de producto; direccionamiento estratégico; gestión empresarial; asociaciones campesinas

Código JEL: M10, P25 


\section{加强埃尔坦博市的农民协会向高附加值产品行 业的转换。 \\ 研究课题: 哥伦比亚 考卡省埃尔坦博市与美藤果产品的

$$
\text { 合作。 }
$$

Diana Ximena Sánchez Tróchez"

Institución Universitaria Colegio Mayor del Cauca

dxsanchez@unimayor.edu.co

Orcid: https://orcid.org/0000-0003-0309-0673

Adriana Diago Ortiz

Institución Universitaria Colegio Mayor del Cauca

adiago@unimayor.edu.co

Orcid: http://orcid.org/0000-0003-1150-4792

Wilfred Fabián Rivera Martínez

Corporación Autónoma del Cauca

wilfred.rivera.m@uniautonoma.edu.co

Orcid: https://orcid.org/0000-0003-2888-7929

Laura Beatriz Potes Ordoñez

Fundación Universitaria de Popayán

laura.potes@docente.fup.edu.co

Orcid: https://orcid.org/0000-0002-3654-4726

Marly Viviana Navia Mera

Institución Universitaria Colegio Mayor del Cauca

marly_navia@unimayor.edu.co

Recibido: 29/09/2020; Aceptado: 05/11/2020; Publicado: 13/09/2021

文章摘要

研究目标: 本研究是在以下指导性问题下开展的: 将传统农物转变为高附加值产品的必 要要素是什么? 从理论角度出发, 此研究会根据Schultz (1967) 农业现代化和 Chiavenato $(2017)$ 组织管理提出假设。

" Autor de correspondencia:

dxsanchez@unimayor.edu.co

ISSN 0212-1867 / e-ISSN 1989-3574

(C) ESIC Editorial,

ESIC Business \& Marketing School

https://revistasinvestigacion.esic.edu/esicmarket 
分析方法: 本研究已描述性研究为基础, 根据案例研究的标准, 采用半结构化调查, 应 此调查可以通过对合作周围的活动、流程、人员及潜在市场进行普遍情况、习俗和态度 的了解。

研究结论: 本研究在农业经济学提出了五个要素, 它们会允许社区将传统农业转变为具 有高附加值的产品, 因此提高其竞争力, 并得出结论, 组织需要突出产品属性的战略, 并在战略方向上做出更大的努力。

研究局限: 研究的结果被推断给具有相似特征, 大小和焦点的组织, 包括农业公司和有 兴趣加强农业经济的哥伦比亚合作社。相反, 该分析有助于更深度的了解有关现象, 但 有可能得到那些对农村行业转型感兴趣的组织的否认。但是这些组织不包括来自工业化 行业。

实际应用: 本研究在战略方向和人力资源管理领域的用途较大, 因此允许机构操作可视 化产品创新计划。

关键词：农村经济；产品创新；战略方向；企业管理；农会

JEL 分类号：M10, P25 


\section{Introducción}

La sociedad en general está en constante transformación, expuesta a diversos desarrollos y avances tecnológicos, científicos, culturales, que fomentan permanentemente una visión de cambio para convertirse en actores claves de productividad. Este nivel de exigencia y competencia hace que toda organización tome acciones de direccionamiento estratégico que le permita identificar productos que se adapten a mercados poco explorados, considerando el entorno, su impacto social, así como la demanda potencial y real del sector en el que está inmerso. Sin embargo, es necesario mencionar que el campo colombiano y en especial, en regiones intermedias, las comunidades rurales siguen practicando la agricultura tradicional a partir de métodos extractivos y poco sostenibles.

Del mismo modo, en los últimos años se han venido consolidando iniciativas que hacen frente a esta trasformación productiva como son los negocios inclusivos cuyo propósito es la evolución de las necesidades y carencias en oportunidades sostenibles, replicables y escalables, siendo una de las innovadoras estrategias empresariales que fomentan el trabajo articulado, fortalecen las cadenas de valor y proponen una manera diferente de abordar los modelos de negocio (Ángel Cano, 2013).

Theodore William Schultz en 1967 plantea una posible estrategia para conseguir este objetivo de transformación productiva mediante economías agrarias, que permiten a las comunidades del sector rural una adecuada transformación de cultivos tradicionales hacia frutos de alto valor agregado, lícitos, visionarios y útiles, incrementando la productividad, haciéndolos más competitivos y proporcionándoles un posicionamiento en el mercado (Steenkamp et al., 2021).

En ese orden de ideas, la teoría de la Pirámide de Prahalad plantea como enfoque esencial la necesidad de dejar de ver a los pobres como víctimas o como una carga, y comenzarlos a reconocer como personas persistentes. A partir de este planteamiento, se evidencia que existen posibilidades de crecimiento e innovación para el sector rural colombiano y por supuesto, para regiones en condiciones similares (Hamel \& Prahalad, 2018).

Ahora bien, el Tambo, ubicado en Colombia, específicamente en el departamento del Cauca, a 33 kilómetros de Popayán, capital del departamento, cuenta con una población estimada de 45.804 habitantes (Ministerio del Interior y de Justicia, 2009). Es uno de los municipios más grandes de la región, eminentemente agrícola y forestal que cuenta con gran cantidad de recursos hídricos (tres grandes cuencas: Patía, Cauca y Micay) (Consejo Municipal del Tambo, 2020; Obando, 2013).

Es una región, al igual que el Cauca y Colombia, supremamente diversa en su estructura física, geográfica, demográfica y en su desarrollo poblacional y cultural. (Consejo Municipal del Tambo, 2020). Ha sido fuertemente afectada por conflictos surgidos, en la gran mayoría, como consecuencia de bajos ingresos económicos, ausencia de oportunidades laborales para progresar digna y lícitamente. Pese a ello, han sido varias las iniciativas en materia de desarrollo rural propuestas. 
Por lo anterior, la problemática principal que aborda el presente artículo es la poca innovación en procesos de direccionamiento estratégico con impacto en la cadena de valor del sector rural; para estudiar este fenómeno se analizan las dinámicas de iniciativas rurales como la cooperativa caucana SachaTambo, adscrita a SachaColombia (hoy SumaSach'a), siendo un Ecosistema Empresarial Agroindustrial Inclusivo en Red para agricultores interesados en la industria, convirtiéndose, además, en una comercializadora internacional. En consonancia, desde la teoría de la modernización de la agricultura planteada por Theodore William Schultz en 1967, es pertinente considerar que no solo la tierra y el capital de trabajo son suficientes para transformar cultivos con alto valor agregado, sino que se necesita de otros elementos de gestión que complementen y apalanquen los esfuerzos de direccionamiento estratégico que se vienen dando (Steenkamp et al., 2021).

Las anteriores afirmaciones, contribuyen al propósito de la investigación, a fin de establecer los lineamientos del direccionamiento estratégico a partir de la identificación de variables estratégicas para la Cooperativa SachaTambo según su propio contexto. Esta organización ha logrado un incremento en el número de familias asociadas y la generación de alternativas de producción amigables con el medio ambiente; a su vez, se destaca el interés de la comunidad por llevar el producto y sus derivados a un mayor nivel de conocimiento social.

El artículo se presenta bajo seis apartados. El primero, relaciona a Sacha Inchi, como cultivo de alto valor agregado, en el que se conceptualiza las propiedades del fruto y la forma de trabajo de las cooperativas asociadas. Como segundo aspecto, se presentan los postulados Teóricos, apoyados principalmente en la teoría de la Modernización de la Agricultura planteada por (Steenkamp et al., 2021) con base en los postulados de Schultz (1967) y demás investigadores relacionados con la temática. En seguida, se desarrolla como tercer componente la metodología, de tipo descriptiva y corte transversal, con encuestas semiestructuradas como instrumento de recolección de datos. El documento aborda como cuarto aspecto el desarrollo del trabajo de campo y análisis de resultados, valorando el mercado potencial y los factores asociados a la productividad desde herramientas como las Cinco Fuerzas de Porter y la Matriz de Evaluación de los Factores Externos e Internos. El quinto componente, enfoca variables estratégicas propuestas como: gestión promocional, fortalecimiento de comunidades cooperantes, gestión de procesos de red, gestión del Talento Humano y canales de comercialización desde la cadena de valor. Así mismo, incluye las estrategias de gestión que apoyan la plataforma de direccionamiento estratégico. Finalmente, se presenta el sexto apartado orientado a la discusión y conclusiones que surgen de los resultados obtenidos del estudio.

\section{Sacha Inchi, cultivo de alto valor agregado}

El Sacha Inchi (Plukenetia volúbilis), conocida como la semilla milenaria, es una planta de origen amazónico, también nombrada como Maní Jíbaro o Maní del 
monte. Está distribuido en el trópico latinoamericano, específicamente en el sur de México, Indias Occidentales, Amazonía y Bolivia (López et al., 2001).

Actualmente se ha convertido en un cultivo no tradicional promisorio con un ciclo productivo de 5 a 7 meses, con posibilidades de industrialización, alto potencial de comercialización en mercados nacionales e internacionales (Sachacolombia, 2017). Además, posee un rendimiento económico que ayuda a mitigar los efectos del conflicto y reemplazar, en alguna medida, los cultivos ilícitos que por décadas han ocasionado problemas de tipo social, económico y ambiental (Wang et al., 2018).

Así mismo, la semilla tiene una composición especial que la hace muy apetecida en las industrias nutricionales, cosméticas y medicinales, debido a que la almendra cuenta con altos contenidos de proteínas, aminoácidos, ácidos grasos esenciales (Omegas 3, 6, y 9) y vitaminas, en comparación con otras semillas oleaginosas. Contiene entre un $25 \%$ y $33 \%$ de proteína según la variedad (Aranda-Ventura et al., 2019).

Este modelo agroindustrial constituye una alternativa para la sustitución de cultivos de uso ilícito. De hecho, por la diversidad que propician los pisos térmicos en Colombia y por encontrarse en esta zona de la línea del Ecuador, el Sacha Inchi se ha consolidado como un cultivo no tradicional con potencial para Colombia; según el Ministerio de Agricultura y Desarrollo Rural, el área sembrada en el territorio nacional ha aumentado un $300 \%$ durante los últimos tres años (Bustamante y Muñoz, 2017).

Entre los diferentes métodos empleados, se encuentra el prensado en frio, proceso mecánico mediante el cual, se extrae el aceite de la semilla, libre de químicos. De esta manera, la semilla previamente limpiada, se lleva al cilindro extractor, se somete a presión lentamente hasta alcanzar la temperatura ambiente, logrando el aceite que se empaca en galones, y se decanta. Luego mediante una maquina filtradora se procede a embotellarse.

Este modelo cuenta con el compromiso y apoyo de un grupo de empresas como Aliados Agroindustriales y Comerciales (AAC), que capacitan e impulsan cada uno de los grupos de la red de valor, desde la siembra hasta la comercialización de los productos.

Mediante un estudio cualitativo, fue posible determinar las variables estratégicas enfocadas en atraer inversiones que generen encadenamientos productivos y dinamicen el campo, de tal manera que se impulse la gestión y transformación agroindustrial, logrando que el Cauca y regiones similares sean una puerta de ingreso económico, existiendo hoy en día cerca de 30 hectáreas establecidas en los diferentes puntos cardinales de la región del Cauca.

Pese a las facilidades agronómicas para la obtención de la almendra, se hace evidente un insuficiente aprovechamiento del potencial agroindustrial, debido a los bajos niveles tecnológicos, la ausencia de capacitación de las personas a nivel individual y social.

En razón a la situación expuesta, se crea SachaTambo, organización que promueve la seguridad alimentaria por medio del cultivo y la industrialización de 
Sacha Inchi, estimulando la formación integral de los asociados y sus familias a través de las buenas prácticas agrícolas, la conservación del medio ambiente, incentivando a que se conviertan en socios de la Cooperativa, se empoderen del campo, lo vuelvan más productivo y competitivo.

Como un ejercicio de fortalecimiento en red, "Sachatambo" se asoció a "SumaSach'a", buscando fortalecerse y expandirse como negocio inclusivo, que aporta desarrollo social y crecimiento económico a la comunidad Tambeña, de manera innovadora y sostenible.

Con ello, este producto y la cooperativa Sachatambo, constituyen un referente para otras regiones interesadas en rescatar las prácticas ancestrales, como es el caso del cultivo y beneficios del Sacha Inchi. Son estos proyectos innovadores y la fuerte tendencia al avance y cambio de la sociedad, los que generan la necesidad de priorizar variables estratégicas para implementar de manera sostenible una gestión empresarial hacia modelos de negocio bajo la figura de asociatividad o cooperativismo en el sector rural.

\section{Postulados teóricos}

La investigación se apoyó en una serie de postulados para la estructuración de los lineamientos estratégicos acordes al contexto rural de la cooperativa Sacha Inchi, tomando como principal referente al autor Theodore William Schultz y su teoría de la Modernización de la Agricultura, la cual se enfoca en la importancia que conlleva para las naciones la transformación de la economía rural como una fuente importante de ingresos para la sociedad, y como la tierra y el capital de trabajo no se constituyen en los únicos elementos para lograr el éxito empresarial. Es decir, se requiere, promover el conocimiento, habilidades y destrezas de las comunidades campesinas y demás actores para aprovechar los recursos existentes y potencializar la actividad agrícola (Steenkamp et al., 2021).

La demanda de productos agrícolas está sufriendo cambios sin precedentes, ha incrementado requerimientos de materias primas de alto valor, de productos procesados y preparados. Entonces, las perspectivas de crecimiento constante de alimentos con valor añadido son un incentivo para el desarrollo de las agroindustrias. Serán sostenibles si son competitivas en términos de costos, precios, eficiencia operativa y otros parámetros asociados (Arango et al., 2020).

Por esto, las empresas deben evaluarse para dar dirección a las estrategias y garantizar su supervivencia. La "administración estratégica", por tanto, constituye una herramienta que permite a las organizaciones enfrentar el futuro de manera más previsible, ayudando a orientar los esfuerzos hacia metas realistas de desempeño. (Estrada et al., 2017). Es también, elemento fundamental en cuanto al "diseño organizacional" como proceso para construir y adaptar continuamente la estructura de la organización." (Chiavenato, 2017). 
Así mismo, es importante considerar las tres etapas propuestas por David (2013): la formulación, la implantación y la evaluación de la estrategia, enfocándose en tres fases, la revisión de los factores externos e internos en que se basan las estrategias actuales, la medición del rendimiento y la toma de medidas correctivas

Por consiguiente, el diseño e implementación de procesos de direccionamiento estratégico, requieren tomar las bases de las disciplinas asociadas al tópico en cuestión, pero no son funcionales, sino que se adaptan a las dinámicas propias del contexto, considerando con ello, sus particularidades en razón a las necesidades y expectativas del entorno. En aspectos prácticos, para lograr el objetivo del presente artículo, fue necesario desde un ejercicio colectivo identificar variables necesarias para la plataforma estratégica, entre las que cabe mencionar la gestión empresarial, entendida como la evolución de nuevas sociedades de información y conocimiento, que implica una visión global para el desarrollo de nuevas estrategias hacia la permanencia en el mercado, la satisfacción de los clientes y la sostenibilidad (Pitre et al., 2020).

Así mismo, se reconoció como un aspecto determinante el fortalecimiento de comunidades participantes, desde una visión de cooperativismo, incluyendo la necesidad del trabajo en equipo con fines solidarios, de equidad y justicia social (Rivera y Labrador, 2013).

Como tercer elemento, se determinó que la gestión de procesos de red facilita la contribución y genera un impacto mayor que el trabajo individual, entre otros aspectos, por la transferencia de conocimientos, el aumento del capital de trabajo, y la capacidad productiva (Ayaviri et al., 2017).

El cuarto elemento abordado es la gestión del talento humano, precisamente por el enfoque teórico de Theodore William Schultz, quien resalta que no es suficiente la tierra y el capital de trabajo para hacer productiva la actividad agrícola, se requiere del desarrollo de habilidades y técnicas que permitan de forma articulada considerar todos los agentes externos e internos asociados a la actividad para lograr resultados de alto impacto, lo que no es posible si no se considera el factor humano como determinante en el proceso. En este caso, la gestión del talento humano es primordial por la connotación que tiene el crecimiento de familias asociadas a la cooperativa, el crecimiento personal y laboral que significa para el equipo hacer parte de la cooperativa (Chiavenato, 2017).

Finalmente, el último elemento necesario a considerar como eje principal en el direccionamiento estratégico para el caso en cuestión, son los canales de comercialización desde la cadena de valor, debido que la organización puede llevar sus productos a los clientes de la forma más eficiente y efectiva, considerando que la producción está garantizada en la mayoría de los casos, pero su venta no. (Rogel y Yumaria, 2019) 


\section{Metodología}

Esta investigación pretende identificar de forma precisa y directa aquellos aspectos que son primordiales para conocer y aportar al análisis interno y externo de la Cooperativa SachaTambo, además de identificar mercados alternativos que permitan incrementar la comercialización de los derivados del fruto Sacha Inchi, en especial el aceite, al ser un punto de referencia para otras organizaciones de sectores similares.

Así, la investigación descriptiva constituye el tipo de investigación de mayor conveniencia para describir la población, situación o fenómeno alrededor del cual se centra el estudio. Procura brindar información acerca del qué, cómo, cuándo y dónde relativo al problema de investigación, sin darle prioridad a responder al "por qué" ocurre dicho problema. Como dice su propio nombre, esta forma de investigar "describe", no explica (Hernández, 2018). Desde la perspectiva del método, el estudio de caso se manifiesta como el método de investigación por antonomasia en esta lógica descriptiva. Esta herramienta permitió recoger las principales variables estratégicas a considerar en una adecuada gestión empresarial de la cooperativa Sacha Tambo (Álvarez, 2009)

La investigación descriptiva, facilitó la recolección de datos, para su posterior análisis, basados en conocer las situaciones, costumbres y actitudes predominantes a través de la descripción exacta de las actividades, procesos y personas del entorno de la Cooperativa, así como su mercado potencial, ejercicio que contó con la participación del equipo administrativo de la organización y las familias asociadas.

Las fuentes directas se abordaron por medio de una encuesta semiestructurada, elaborada de acuerdo con los criterios de caracterización de los productos. Esto permitió validar la información obtenida por fuentes secundarias. En relación con las fuentes indirectas, se sistematizaron por medio de cuadros o matrices, empleando indicadores de estadística descriptiva para apoyar las observaciones de campo, las opiniones de los expertos y del equipo de trabajo, respecto al diagnóstico de los datos recolectados.

Conviene destacar que, en el trabajo de campo, la recolección de la información se hizo a través de dos poblaciones objeto de estudio. La primera conformada por los consumidores potenciales de estratos socioeconómicos 4, 5 y 6 de la ciudad de Popayán, capital del departamento del Cauca. Este grupo poblacional presenta mayor interés por la adquisición de productos de base orgánica, por lo que se pretendió identificar su interés en el consumo del aceite de sacha inchi. Para el estudio se estableció un tamaño de muestra de 164 encuestas con un nivel de confianza del 95\% y un margen de error del $5 \%$, que fueron sistematizadas por medio del programa SPSS. La segunda población fue la comunidad SachaTambo, conformada por 140 familias asociadas debidamente encuestadas, dada la importancia que tiene el trabajo en Red y la incidencia para el cumplimiento de los objetivos organizacionales. Con ellos se abordaron elementos desde la lectura del contexto encontrando o evidenciando las variables que luego se trabajan más adelante. 


\section{Desarrollo del trabajo de campo y análisis de los resultados}

En esta sesión se describen los resultados de la investigación a partir del estudio cualitativo realizado por los investigadores. Se divide en 2 sub sesiones según los grupos abordados. En la primera sesión, se analizan los resultados obtenidos del mercado potencial, puntualizando en aspectos demográficos, gustos y preferencias. Con ello, se pretende comprender elementos de percepción que permite para este tipo de ejercicios considerar variables determinantes en el diseño de estrategias de promoción y comercialización, con el fin de tomar decisiones coherentes de la imagen que se quiere transmitir, enfocada a un producto orgánico, producido en el campo colombiano y por familias campesinas bajo sistema de cooperativismo. En una segunda sesión, se abarcan elementos relacionados con el funcionamiento y gestión de la cooperativa según información de las familias asociadas y su representante legal.

\subsection{Características, preferencias y frecuencia de compra del mercado potencial}

El principal factor que se debe tener en cuenta en la identificación del mercado potencial es el conocimiento de las características específicas del mismo, como preferencias, frecuencia de compra, capacidad de pago y afinidad hacia el producto.

Se pudo evidenciar que la población objeto de estudio, conformada en un $62 \%$ por hombres y un $38 \%$ por mujeres, de edades promedio entre 30 y 41 años (33\%), 18 y 29 años (30\%) y mayores de 42 años (37\%), perciben ingresos promedios mensuales entre 1.8 a 2.7 Salarios Mínimos legales Vigentes, siendo el SMLV de $\$ 877.802$ pesos para el caso colombiano 2020 .

Ahora bien, este tipo de productos se asocian con la nueva tendencia de snackficación, fenómeno que transforma la comida tradicional en un formato más amigable y práctico, generando oportunidades de mercado. Entre sus principales beneficios se encuentran los aportes y percepción de los consumidores hacia el cuidado de la salud (Jodar Marco, 2018).

De esta manera, el $67 \%$ de los encuestados indicaron que perciben como positivo para su salud consumir este alimento porque aporta a su nutrición, mientras que un $33 \%$ expresan que su consumo está asociado más a una preferencia de gusto que de cuidado, resultado que puede darse por la falta de conocimiento de sus propiedades.

Otra de las variables necesarias para la adecuada comercialización del producto radica en el conocimiento de la frecuencia de compra de productos saludables relacionados con snacks, comportamiento que pudo verificarse con la investigación, encontrando que las personas cada vez más están conscientes del cuidando de su alimentación, su cuerpo y por ende de su salud. Se observa que el $42 \%$ de encuestados expresaron cuidarse a diario, consumiendo alimentos saludables, dato primordial para potencializar el conocimiento y distribución de estos productos, teniendo en cuenta los beneficios mencionados del Sacha Inchi y sus derivados especialmente el aceite. 
Existen entonces características principales que prevalecen para los consumidores y que deben ser tenidas en cuenta para su adecuada promoción. Entre tanto, y respecto a las características que predominan al momento de elegir un producto alimenticio, están los complementos nutritivos con un $42 \%$, el precio en un $28 \%$ y un $30 \%$ entre presentación y calidad. Estos factores permiten a las marcas encaminadas hacia productos saludables mayor grado de aceptación por el mercado, como es el caso de Sachatambo.

Sin embargo, también se requiere analizar el conocimiento del público objetivo frente a las propiedades nutritivas, uso y beneficios del Sacha Inchi. Al respecto, se pudo determinar que un $60 \%$ de la población encuestada desconoce este fruto milenario. No obstante, dentro de las estrategias a desarrollar para productos innovadores de alto valor agregado como este, se encuentra el diseño de mecanismos que fomenten, difundan, y formen el producto en el mercado potencial, logrando con ello el aprovechamiento de esta semilla, no solo desde sus beneficios para la salud y la belleza, sino también como una manera de apoyar la producción nacional del sector rural.

Así mismo, fue necesario determinar si el público potencial conocía productos que contengan el fruto, encontrando que un $72 \%$ si lo reconoce, aunque no se especifica en qué tipo de presentaciones. De igual forma, el $93 \%$ de los encuestados expresaron que adquieren productos que aportan salud, belleza y bienestar al mismo tiempo.

En lo que respecta al precio a pagar por adquirir estos productos, un 53\% respondió que entre $\$ 40.000$ y $\$ 53.000$ pesos colombianos, seguido de un $22 \%$ más de $\$ 54.000$ pesos colombianos y un $24 \%$ pagaría menos de $\$ 39.000$ pesos colombianos según presentaciones y gramaje del producto, lo que permitiría a la Cooperativa SachaTambo y marcas de sectores similares posibilidades importantes para el desarrollo del consumo de esta semilla y sus derivados, encontrando, una valoración económica por los productos que integran o contienen la mayor cantidad de beneficios.

En relación con el lugar o lugares que les gustaría adquirir estos productos, el $80 \%$ de los encuestados prefieren encontrarlos en supermercados e internet, mientras que en un $20 \%$, lo prefieren en tiendas especializadas.

Ahora bien, respecto a la presencia de sustitutos percibidos por la población objeto de estudio, el $76 \%$ respondió que sí conoce, sin embargo, para el cuidado y la belleza de tipo industrial y no de tipo orgánico, lo que evidencia su carácter de sustituto indirecto. Finalmente, los factores más importantes a valorar para que las personas se concienticen y mejoren su alimentación según la población abordada, en un 50\% está relacionado con crear hábitos y estilos de vida saludable, mientras que el otro $50 \%$, expresan la necesidad de incrementar la educación en la alimentación y nutrición. 


\subsection{Factores asociados a la productividad desde las cinco fuerzas de Porter}

El análisis de las cinco fuerzas de Porter tiene un enfoque analítico para recopilar información importante frente al diseño del plan estratégico, facilitando el desarrollo de una estrategia para competir en mejores condiciones. Este análisis vigila los factores que influyen en la rentabilidad del negocio a largo plazo, así como los elementos que tienen un impacto directo en la organización y por ende en la rentabilidad.

Es decir, brinda información sobre las tendencias del mercado con el objetivo de identificar oportunidades, conocer el entorno de la empresa para anticipar acciones y reconocer la ventaja competitiva (Jaya y Yuliarmi, 2019).

F1. Poder de negociación de los clientes: para la Cooperativa, el reconocimiento de la semilla de Sacha Inchi ha sido un pilar fundamental para asociar a más agricultores y conseguir nuevos clientes, a medida que surgen nuevas unidades empresariales. Estas hacen que los cultivos se masifiquen y estén en constante competitividad. $\mathrm{Al}$ tener más opciones, otorga a los usuarios mayores posibilidades y facilidades de adquirir los productos; los márgenes de beneficio demuestran notablemente el poder de compra y cómo los clientes están dispuestos a pagar por productos de buena calidad a un precio justo en función de su poder de negociación. En la actualidad el mercado de la alimentación saludable, categoría en la cual se encuentra el Sacha Inchi muestra un mayor crecimiento. Según datos de la consultora Nielsen, los productos saludables representan el $7 \%$ de la canasta familiar en Colombia y sus ventas han aumentado un $12 \%$ en el último año. Se observa entonces que el interés por cuidarse a través de la alimentación saludable es más que una moda y se ha convertido en una gran macrotendencia de consumo en el mundo (Fmcg y Retail, 2018).

F2. Poder de negociación de los proveedores: SachaTambo cuenta con un alto poder de negociación de proveedores, debido a que SachaColombia apoya a las Unidades Empresariales con aliados como BioAzul, Biorrefinería, InnovaSemillas, SachaFormadores SAS, SumaSacha, Proalco, SachaConsultoría, Soluciones microbianas del trópico, Del Monte Agronegocios inclusivos, universidad de Santander y logyca, lo que les facilita trabajar en conjunto con especialistas en todo los temas y requerimientos que abarca el cultivo de Sacha. Por este motivo, los proveedores de esta industria se consideran relativamente fuertes. Es uno de los factores que le otorga a la cooperativa una gran fortaleza ya que se apoya del trabajo en red para la transferencia de tecnología en el cultivo y en general en la cadena productiva del Sacha Inchi.

F3. Amenaza de los nuevos competidores: Dentro del mercado, se identifican emprendedores que buscan la formación y creación de estos negocios de forma independiente a la Cooperativa, sin tener en cuenta los procesos que se han gestado para garantizar un producto de alta calidad; además existen fuertes barreras de entrada para los competidores, debido a los múltiples beneficios que otros productos no logran suplir, sin embargo, depende de su encadenamiento productivo, su impacto ambiental y social. Este factor otorga una ventaja competitiva relacionado con el 
impacto de la empresa en el entorno. Si bien hay una gran cantidad de competidores, estos lo hacen de manera independiente, mientras que la cooperativa Sacha Inchi cumple una labor social, factor que se convierte en una diferenciación en el mercado y en un aspecto favorable en la decisión de compra.

F4. Amenaza de productos sustitutivos. El aceite de Sacha Inchi está en la categoría de aceites funcionales; entre los principales productos similares y sustitutos de Sacha Inchi se encuentran el aceite de oliva, aceite de aguacate y el aceite de coco. Si bien estos aceites ofrecen beneficios para la salud, el aceite de Sacha Inchi es altamente nutritivo debido a la cantidad de proteínas que es de $33 \%$ y su alto índice de ácidos grasos omega 3 y 6 con un porcentaje de $48.6 \%$ y 36,8\% respectivamente, lo que lo convierte en el mejor aceite para consumo humano doméstico, ya que refuerza el sistema inmunológico y retarda la llegada de la glucosa a la sangre. En consecuencia, el Sacha Inchi comparativamente cuenta con propiedades superiores que otros productos sustitutos, logrando con ello, proporcionar beneficios importantes para una dieta complementaria (Gómez y Montaña, 2019).

F5. Rivalidad entre los competidores existentes. En el mercado en el que se encuentra la Cooperativa, la rivalidad de los competidores es significativa, en cuanto a los precios que manejan, pues en su gran mayoría, las otras empresas no hacen uso de la maquinaria y tecnología que la transformación de la semilla requiere, lo que les facilita tener unos precios por debajo de la Cooperativa SachaTambo. La tabla 1 evidencia la posición en la que se encuentra con respecto a otros productos sustitutos.

Se observa que el producto cuenta con las herramientas necesarias para un buen reconocimiento en el sector, encontrando como fortalezas, su política de precios, la variedad de productos, el uso adecuado de maquinaria y equipos, garantizando procesos eficientes, eficaces, y finalmente preservando el medio ambiente al generar productos ecológicos y acordes con el cuidado del entorno. Sin embargo, dada su juventud, requiere fortalecer acciones de publicidad e incrementar su nivel de reconocimiento en el mercado.

Tabla 1. Análisis de la Competencia.

\begin{tabular}{lllll}
\hline $\begin{array}{l}\text { EMPRESA } \\
\text { CONCEPTO }\end{array}$ & $\begin{array}{l}\text { ACEITE DE SACHA } \\
\text { INCHI }\end{array}$ & $\begin{array}{l}\text { ACEITE DE } \\
\text { OLIVA }\end{array}$ & $\begin{array}{l}\text { ACEITE DE } \\
\text { PESCADO }\end{array}$ & $\begin{array}{l}\text { ACEITE DE } \\
\text { COCO }\end{array}$ \\
\hline Publicidad & Debilidad & Fortaleza & Fortaleza & Debilidad \\
\hline Reconocimiento & Debilidad & Fortaleza & Fortaleza & Fortaleza \\
\hline Política de precios & Fortaleza & Fortaleza & Debilidad & Fortaleza \\
\hline Variedad en productos & Fortaleza & Debilidad & Debilidad & Debilidad \\
\hline Maquinaria y equipos & Fortaleza & Fortaleza & Debilidad & Fortaleza \\
\hline $\begin{array}{l}\text { Preservación del medio } \\
\text { ambiente }\end{array}$ & Fortaleza & Fortaleza & Debilidad & Fortaleza \\
\hline
\end{tabular}

Fuente: Elaboración Propia. 


\subsection{Factores asociados al entorno externo e interno}

EFE. Matriz de Evaluación de los Factores Externos. Se identificaron oportunidades y amenazas por parte del entorno, logrando determinar con ello cuáles deben ser las decisiones más acertadas para cada situación en relación con el producto y la organización como se observa en las tablas 2 y 3.

Tabla 2. Tabla de Calificación.

\begin{tabular}{ll}
\hline TABLA CALIFICACIÓN & \\
\hline 1 & AMENAZA MAYOR \\
\hline 2 & AMENAZA MENOR \\
\hline 3 & OPORTUNIDAD MENOR \\
\hline 4 & OPORTUNIDAD MAYOR \\
\hline
\end{tabular}

Fuente: Elaboración Propia.

Tabla 3. Matriz de Evaluación de Factor Externo (EFE).

\begin{tabular}{llll}
\hline VARIABLES EFE & & & \\
\hline Oportunidades & Pond. & Clasificación & Result. Pond. \\
\hline Inversión en equipos de última tecnología & 0,07 & 3 & 0,28 \\
Empleados comprometidos con la organización & 0,06 & 4 & 0,18 \\
Reconocimiento en el mercado & 0,09 & 3 & 0,36 \\
Acceso a nuevos mercados & 0,10 & 4 & 0,4 \\
Responsabilidad social & 0,11 & 4 & 0,33 \\
$\begin{array}{l}\text { Mejoramiento del estilo de vida } \\
\text { Calidad de los productos }\end{array}$ & 0,10 & 3 & 0,4 \\
\hline SUBTOTAL OPORTUNIDADES & 0,06 & 4 & 0 \\
\hline Amenazas & 0,59 & & 1,95 \\
\hline $\begin{array}{l}\text { Duplicidad del aceite con menor calidad } \\
\text { Competidores con fuertes estrategias }\end{array}$ & 0,11 & 1 & 0,11 \\
publicitarias & 0,10 & 2 & 0,2 \\
$\begin{array}{l}\text { Aumento de la inflación, reflejando disminución } \\
\text { en el poder adquisitivo }\end{array}$ & 0,06 & 2 & 0,12 \\
$\begin{array}{l}\text { Competencia con precios más económicos } \\
\text { Las otras empresas de los sustitutos tienen }\end{array}$ & 0,08 & 1 & 0,08 \\
reconocimiento a nivel regional y nacional & 0,06 & 2 & 0,12 \\
\hline SUBTOTAL AMENAZAS & 0,41 & & 0,63 \\
\hline TOTAL & $\mathbf{1 , 0 0}$ & $\mathbf{2 , 5 8}$ & \\
\hline
\end{tabular}

Fuente: Elaboración Propia. 
EFI. Matriz de Evaluación de los Factores Internos. Fue posible evaluar información económica, social, cultural, demográfica, ambiental, política, gubernamental, jurídica, tecnológica y competitiva, con el fin de fortalecer a la organización frente a sus competidores, evidenciada en la tabla 4.

Tabla 4. Matriz de Evaluación de Factor Interno (EFI).

\begin{tabular}{llll}
\hline VARIABLES EFI & & & \\
\hline FORTALEZAS & PESO & CALIFICACION & VALOR POND. \\
\hline $\begin{array}{l}\text { Buena prestación de servicio tanto a socios } \\
\text { como clientes }\end{array}$ & 0,11 & 4 & 0,44 \\
$\begin{array}{l}\text { Requisitos mínimos de seguridad en la } \\
\text { Cooperativa }\end{array}$ & 0,08 & 3 & 0,24 \\
$\begin{array}{l}\text { Adecuada infraestructura } \\
\text { Personal idóneo para brindar el servicio }\end{array}$ & 0,09 & 4 & 0,36 \\
$\begin{array}{l}\text { Variedad en los productos y con altos } \\
\text { estándares de calidad }\end{array}$ & 0,06 & 4 & 0,24 \\
$\begin{array}{l}\text { Fidelización de los clientes } \\
\text { Ubicación de un punto visible, asequible y seguro } \\
\text { para los clientes. }\end{array}$ & 0,09 & 3 & 0,36 \\
\hline SUBTOTAL FORTALEZAS & 0,07 & 3 & 0,21 \\
\hline DEBILIDADES & 0,59 & & 0,27 \\
\hline $\begin{array}{l}\text { Tecnología apta de la maquinaria } \\
\text { Bajas estrategias de ventas }\end{array}$ & 0,09 & 2 & 2,12 \\
$\begin{array}{l}\text { Falta de un portafolio de servicios } \\
\text { Competencia informal }\end{array}$ & 0,04 & 2 & 0,18 \\
$\begin{array}{l}\text { Otorgar descuentos y promociones } \\
\text { Baja publicidad }\end{array}$ & 0,05 & 2 & 0,08 \\
$\begin{array}{l}\text { Falta de planificación administrativa, pues } \\
\text { no cuenta con una estructura organizacional } \\
\text { definida. }\end{array}$ & 0,06 & 1 & 0,1 \\
\hline SUBTOTAL DEBILIDADES & 0,04 & 2 & 0,06 \\
\hline TOTAL, EFI & 1 & 2 & 0,08 \\
\hline & & & 0,1 \\
\hline
\end{tabular}

Fuente: Elaboración Propia.

Como se observa en la anterior tabla, el peso ponderado entre fortalezas y debilidades alcanzan una sumatoria de 2,8, de las cuales las fortalezas representan el 2,12 y están por encima de las debilidades, que obtiene una suma de 0,68 . Esto que indica que si se logra dar mayor apoyo y dedicación a las fortalezas, se podrán superar con mayor eficacia las debilidades y así poder proyectar la Cooperativa a que sea reconocida en el mercado tanto regional como Nacional. 
BCG. Análisis de Crecimiento - Participación: fue posible priorizar recursos entre distintas áreas de negocios o Unidades Estratégicas de Análisis. La matriz Bostón Consulting Gtopu-BCG presentada en la tabla 5 permite clasificar el portafolio de la cooperativa considerando sus flujos de caja y crecimiento, prevaleciendo los productos como estrellas, vacas, perros o interrogantes según la tasa de crecimiento y la participación del mercado (Arias Mesía, 2013).

Tabla 5. Análisis de Crecimiento (BCG).

\begin{tabular}{ll}
\hline Estrella & Dilema \\
$\begin{array}{l}\text { Aceite de Sacha Inchi } \\
\text { X 250ml. }\end{array}$ & Cerveza de Sacha Inchi \\
\hline Vaca & Perro \\
Snacks de dulce, sal & Torta Proteica \\
Achocolatados y de sal-limón & Harina de Sacha Inchi \\
\hline
\end{tabular}

Fuente: Elaboración Propia.

Como lo indica la tabla anterior, se puede decir que SachaTambo tiene dominio sobre el mercado con su producto estrella, el aceite de Sacha Inchi en presentación x $250 \mathrm{ml}$, siendo el que origina mayores ingresos.

FODA: Mediante esta matriz se identificaron aspectos relevantes a partir de información obtenida por la comunidad de la cooperativa que se expone a continuación en la tabla 6.

Variables Estratégicas: tomando como referencia los factores asociados a la productividad y demás elementos analizados, se presentan las variables estratégicas más representativas para el fruto Sacha Inchi, cuya cadena productiva y de comercialización se hace a través de trabajo en red bajo la figura de cooperativa presentada a continuación en la tabla 7.

Estrategias de gestión: Como complemento de las anteriores, se presenta en la tabla 8, una agrupación estratégica de gestión que permite abordar seis frentes principales de las organizaciones de este tipo. 
Tabla 6. Matriz FODA.

\begin{tabular}{|c|c|c|}
\hline & Oportunidades. & Amenazas \\
\hline $\begin{array}{l}\text { Matriz FODA } \\
\text { de Cooperativa Sachatambo }\end{array}$ & $\begin{array}{l}\text { 1. Inversión en equipos de última } \\
\text { tecnología. } \\
\text { 2. Amplios mercados para } \\
\text { incursionar. } \\
\text { 3. Reconocimiento en el mercado } \\
\text { por su muy alta calidad. } \\
\text { 4. Aporte al cuidado y } \\
\text { preservación del medio } \\
\text { ambiente. } \\
\text { 5. Usuarios dispuestos a pagar } \\
\text { por un buen servicio. } \\
\text { 6. Buena relación con los clientes. } \\
\text { 7. Poco desarrollo del producto } \\
\text { en el mercado y poca } \\
\text { diversificación. } \\
\text { 8. Tendencia a nivel mundial } \\
\text { por el consumo de productos } \\
\text { naturales. } \\
\text { 9. Aumento en las exportaciones } \\
\text { y países de destino. }\end{array}$ & $\begin{array}{l}\text { 1. Pérdida de clientes. } \\
\text { 2. Aparición de nuevas } \\
\text { Cooperativas. } \\
\text { 3. Competidores con fuertes } \\
\text { estrategias publicitarias. } \\
\text { 4. Competencia con precios } \\
\text { más económicos. } \\
\text { 5. Competencia informal. } \\
\text { 6. Presencia de servicios } \\
\text { sustitutos. } \\
\text { 7. Número de concentración } \\
\text { de proveedores. }\end{array}$ \\
\hline
\end{tabular}

\begin{tabular}{|c|c|c|}
\hline Fortalezas & Estrategias FO & Estrategias FA \\
\hline $\begin{array}{l}\text { 1. El aceite de Sacha Inchi tiene } \\
\text { propiedades medicinales } \\
\text { y nutritivas por su alto } \\
\text { contenido en omega } 3,6 \text { Y } 9 \text { en } \\
\text { comparación a otros aceites. } \\
\text { 2. Ubicación geográfica } \\
\text { estratégica por sus } \\
\text { condiciones climáticas y de } \\
\text { suelo, que favorece el cultivo } \\
\text { de esta semilla. } \\
\text { 3. Semilla con rápido } \\
\text { crecimiento y de cosecha } \\
\text { orgánica. } \\
\text { 4. Adecuada infraestructura } \\
\text { física: socios con terreno para } \\
\text { el cultivo de esta semilla. } \\
\text { 5. Calidad de productos. } \\
\text { 6. Personal idóneo, con } \\
\text { conocimiento, experiencia y } \\
\text { valores. } \\
\text { 7. Tecnología apta de la } \\
\text { maquinaria. }\end{array}$ & $\begin{array}{l}\text { (O3, F1-F8) Aprovechar la } \\
\text { diversidad de propiedades del } \\
\text { Sacha Inchi y personal capacitado } \\
\text { para generar uno o varios } \\
\text { productos nuevos que satisfagan } \\
\text { la actual tendencia natural. } \\
\text { (O9, F3) Utilizar el rápido } \\
\text { crecimiento de la semilla y su } \\
\text { transformación para potenciar la } \\
\text { incursión en distintos mercados. } \\
\text { (O8, F2) Aprovechar la ubicación } \\
\text { de la Cooperativa para potenciar } \\
\text { el cultivo de la semilla. } \\
\text { (O6, F10) A provechar la } \\
\text { fidelidad de los clientes hacia } \\
\text { la empresa, en la calidad de } \\
\text { los productos y servicios para } \\
\text { aumentar la participación en el } \\
\text { mercado. }\end{array}$ & $\begin{array}{l}\text { (A7, F7) Buscar proveedores } \\
\text { más cercanos para disminuir } \\
\text { costos. } \\
\text { (A4, F5) No enfocarse } \\
\text { únicamente, en la } \\
\text { competencia de los precios, } \\
\text { sino de mantener y mejorar la } \\
\text { calidad de la materia prima y } \\
\text { sus derivados, al igual que los } \\
\text { servicios y la atención a los } \\
\text { clientes. } \\
\text { (A5, F4) Fortalecer y dar a } \\
\text { conocer los puntos a favor } \\
\text { que tiene la Cooperativa } \\
\text { frente a la competencia, para } \\
\text { atraer más clientes. }\end{array}$ \\
\hline
\end{tabular}




\section{Tabla 6. Matriz FODA. (Continuación)}

8. Variedad en productos de calidad.

9. Buena relación con clientes y proveedores.

10. Existencia de información que respalda los usos, beneficios y aplicaciones tradicionales.
(O4, F9) Incentivar a socios, proveedores y comunidad en general al cuidado y preservación del medio ambiente por medio de las buenas prácticas ecológicas.
(A6, F6) Que la buena relación con los clientes, asociados y proveedores sirvan como referencia, para que sean ellos los portadores de publicidad indirecta.

Debilidades
1. Pocas estrategias de ventas.
2. Carencia de un portafolio de
servicios.
3. Falta descuentos y
promociones
4. Escasez de Publicidad.
5. Falta de estructura
organizacional.
organizacional.

\section{Estrategias DO Estrategias DA}

(O5, D3) Incentivar a clientes con descuentos, promociones u ofertas diarias, semanales o mensuales.

(O2, D2) Implementación del portafolio de servicios para realizar convenios o alianzas con empresas.

(O7, D4) Presencia en nuevos canales de comunicación para crear en los clientes recordación de marca.

(O1, D5) Diseño e implementación de la estructura organizacional, para un mejor desempeño dentro de la empresa.
(A1, D1) Crear un buzón de sugerencias para saber cuáles son las necesidades de los clientes y así poder satisfacerlas.

(A3, D4) Crear medios publicitarios y de mercadeo para ser reconocidos por los productos y servicios que se prestan.

(A5, D5) Mejorar el nivel de capacitación de los empleados, para así mejorar la prestación del servicio.

(A2, D1) Dar a conocer a los clientes que la Cooperativa cumple con los requisitos necesarios para prestar un excelente servicio de calidad.

Fuente: elaboración propia

Tabla 7. Variables Estratégicas.

\begin{tabular}{ll}
\hline Objetivos & Estrategias \\
\hline $\begin{array}{l}\text { Incrementar la participación en } \\
\text { el mercado, dando a conocer la } \\
\text { transformación del producto (aceites, } \\
\text { snacks, torta proteica) y fomentar su } \\
\text { consumo. }\end{array}$ & $\begin{array}{l}\text { E1. Gestión promocional: diseñar campañas publicitarias de } \\
\text { todos los productos que se ofertan, aprovechando el rápido } \\
\text { crecimiento de la semilla, su transformación y la diversidad } \\
\text { de sus propiedades y beneficios que posee el Sacha Inchi, }\end{array}$ \\
\hline $\begin{array}{l}\text { la ubicación en la que se dan los cultivos, y aumentar la } \\
\text { incursión en distintos mercados. }\end{array}$ \\
$\begin{array}{l}\text { Empliar la comunidad de socios de } \\
\text { manera sostenible, difundiendo la } \\
\text { importancia del cultivo, generando } \\
\text { conciencia sobre su consumo. }\end{array}$ & $\begin{array}{l}\text { Implementar una plataforma de socios que permita el } \\
\text { sostenimiento para la producción y comercialización del } \\
\text { producto y sus derivados. }\end{array}$ \\
\hline
\end{tabular}


Tabla 7. Variables Estratégicas. (Continuación)

\begin{tabular}{ll}
\hline $\begin{array}{l}\text { Garantizar una estructura } \\
\text { organizacional centrada en el trabajo } \\
\text { colaborativo bajo la figura de } \\
\text { cooperativa }\end{array}$ & $\begin{array}{l}\text { E3. Gestión de procesos de red. Diseñar un manual de } \\
\text { funciones, procedimientos, definición de políticas, derechos } \\
\text { y deberes de los asociados y de personal que actualmente } \\
\text { está vinculado directa e indirectamente al trabajo en red. }\end{array}$ \\
\hline $\begin{array}{l}\text { Fomentar el desarrollo del talento } \\
\text { humano de la comunidad de asociados. }\end{array}$ & $\begin{array}{l}\text { E4. Gestión del Talento Humano: diseñar e implementar } \\
\text { un plan de gestión del talento humano articulado a la } \\
\text { plataforma estratégica de la organización. }\end{array}$ \\
\hline $\begin{array}{l}\text { Determinar los canales de } \\
\text { comercialización del producto } \\
\text { basándose en la cadena de valor }\end{array}$ & $\begin{array}{l}\text { E5. Canales de comercialización desde la cadena de valor: } \\
\text { Diseñar e el portafolio de servicios para realizar convenios o } \\
\text { alianzas empresariales. } \\
\text { Estructurar la cadena de valor del producto y sus derivados } \\
\text { con el fin de focalizar la segmentación de mercado. }\end{array}$ \\
\hline
\end{tabular}

Fuente: Elaboración Propia.

Tabla 8. Estrategias a Implementar.

\begin{tabular}{ll}
\hline Tipo de estrategia & Estrategias a implementar \\
\hline $\begin{array}{l}\text { Estrategia de promoción y y } \\
\text { comunicación }\end{array}$ & $\begin{array}{l}\text { Presencia web del producto y sus derivados, así como de las } \\
\text { organizaciones que los representan (historia, políticas, misión, visión), } \\
\text { permitiendo atraer nuevos clientes, incrementar los socios activos, } \\
\text { generando acercamiento a las comunidades interesadas y población } \\
\text { potencial de consumo. }\end{array}$ \\
\hline $\begin{array}{l}\text { Estrategia de servicio al } \\
\text { cliente y asociados. }\end{array}$ & $\begin{array}{l}\text { Determinar la percepción del cliente y asociados frente a los productos } \\
\text { y servicios que se ofrecen, creando un servicio postventa, por medio de } \\
\text { un seguimiento de llamadas telefónicas, buzón de sugerencias, encuestas } \\
\text { de satisfacción por correo electrónico o personal, identificando los } \\
\text { procesos (distribución, calidad, presentación, atención, precio, tiempos de } \\
\text { entrega y condiciones pactadas), para así tomar decisiones preventivas y } \\
\text { correctivas a mejorar con el fin de obtener una participación importante } \\
\text { en el mercado. }\end{array}$ \\
\hline $\begin{array}{l}\text { Estrategia plan de } \\
\text { referidos }\end{array}$ & $\begin{array}{l}\text { pertalecer la red de socios y clientes, obteniendo beneficios de afiliación, } \\
\text { Estrategia de un equipo y consumo de los productos por medio de la web. }\end{array}$ \\
\hline de ventas & $\begin{array}{l}\text { que permita potencializar y promocionar el trabajo colaborativo, cuya } \\
\text { inversión sea rentable y sus ventas sean incrementadas anualmente }\end{array}$ \\
\hline Estrategia ambiental & $\begin{array}{l}\text { Generar conciencia frente a la conservación y preservación de los recursos } \\
\text { naturales. } \\
\text { Capacitar a la comunidad inmediata frente a la importancia del potencial } \\
\text { que disponen. } \\
\text { Realizar convenio con entidades estatales que permitan el reconocimiento } \\
\text { de esta actividad. }\end{array}$ \\
\hline $\begin{array}{l}\text { Contar con base de datos de proveedores que cumplan con la } \\
\text { normatividad, garantizando la calidad en la materia de prima, } \\
\text { maquinaria con precios competitivos. } \\
\text { Acceder a créditos blandos para asociados con la finalidad de apalancar } \\
\text { los procesos de producción, comercialización y posventa }\end{array}$ \\
\hline
\end{tabular}

Fuente: Elaboración Propia. 


\section{Discusión y conclusiones}

Tal y como ha sido comentado a lo largo de este documento, uno de los propósitos ulteriores de la investigación es el aporte de nuevo conocimiento en el ámbito del fortalecimiento de asociaciones campesinas; en este orden de ideas, la información colectada permitió el desarrollo de ejercicios de planificación estratégica, según los postulados teóricos del management desde una postura orientada por el mercado, que valora las características propias de las comunidades y las formas de articulación con actores de interés.

Lo anterior resulta de interés en el ámbito académico dada la posibilidad de aprender de este ejercicio que reconoce el valor agregado no solo desde las características del producto, sino también desde las particularidades de la historia organizacional, sus procesos y mecanismos de articulación. Los análisis realizados dan cuenta de la imperiosa necesidad de fortalecer los ejercicios de promoción y difusión dado que, los productos cuentan con características importantes y con valores agregados con potencial que el mercado no conoce.

El sector rural se enfrenta a desafíos de gran espectro, que invitan a generar alternativas sostenibles para la erradicación de la pobreza, la violencia y la promoción de la sana convivencia. Para ello, una de las estrategias para promover el trabajo participativo, es a través del sistema de asociados como las cooperativas, encontrando entre los aspectos más relevantes, la focalización de recursos en la concientización y capacitación de los agricultores.

Sin embargo, para lograr la transformación de cultivos con estas características, dirigido a mercados poco explorados, la organización debe fortalecer la gestión de marca en los aspectos identificadores de la marca y en el mensaje que se transmita al mercado objetivo, además de realizar convenios o alianzas empresariales. De igual forma, analizar el entorno interno y externo asociado al producto y sus derivados, con el fin de identificar variables controlables y no controlables.

Así mismo, se pudo evidenciar la necesidad de priorizar acciones en productos con mayores atributos sobre la competencia, y determinar la participación de otros con poca incidencia en el mercado, ya sea por desconocimiento del mismo o por considerarse genérico. Es decir, se requiere hacer más visible este tipo de empresas, mediante la implementación del portafolio de servicios para realizar convenios o alianzas con empresas, desarrollar nuevos canales de comunicación para crear en los clientes recordación de marca, contar con una estructura organizacional funcional, que promueva un desempeño eficiente y eficaz, así como decisiones en la gestión del talento humano enfocado hacia resultados en la calidad del servicio.

Es entonces como el éxito de la gestión empresarial está asociada a la determinación de variables estratégicas. En este caso, el estudio identificó cinco como las más importantes: E1. Gestión promocional, E2. Fortalecimiento de comunidades cooperantes, E3. Gestión de procesos de red, E4. Gestión del Talento Humano y E5. Canales de comercialización desde la cadena de valor. 
También se valoraron estrategias de gestión que apoyan la plataforma de direccionamiento estratégico de la organización y que requiere monitorearse de manera constante. En este caso, las estrategias de primer orden clasificadas con la comunidad, fueron: a) estrategia de promoción y comunicación, b) estrategia de servicio al cliente y asociados, c) estrategia plan de referidos, d) estrategia de un equipo de ventas, e) estrategia ambiental y f) estrategia financiera.

Ahora bien, las herramientas administrativas y de gestión que fueron empleadas en el direccionamiento estratégico de la cooperativa Sachatambo, tienen posibilidad de réplica en organizaciones con características similares, así como en emprendimientos pequeños interesados en transformar el sector rural, considerando que estas comunidades, históricamente han realizado trabajo de asociatividad, pero no han contado con suficientes herramientas administrativas para que sus organizaciones se consoliden.

Con este artículo se abren nuevas líneas de investigación, dado que los productos agrícolas con alto contenido nutricional proponen estudios orientados hacia la construcción de herramientas de desarrollo social y económico en los territorios, por su gran demanda internacional y poco nivel de reconocimiento. Asimismo, son aporte de importancia para la construcción de paz en el país, toda vez, que son alternativas de ingreso y empleo. Por lo anterior, el estudio de procesos colectivos alrededor de productos de esta naturaleza constituye uno de los temas de mayor importancia académica y relevancia social.

\section{Referencias bibliográficas}

Álvarez, J., 2009, Cómo hacer investigación cualitativa: Fundamentos y Metodologías. Editorial Paidos, México.

Ángel Cano, M. E., 2013, Negocio inclusivo: gana-gana sostenible. Encadenamiento productivo: Caso Asociación de Recicladores de Cali. Inclusive business: a sustainable win to win. Integrated production system: the case study of the Association of recycling workers in Cali Entrepr. Ploutos, 3(2), 24-33

Aranda-Ventura, J., Villacrés-Vallejo, J., y Rios-Isern, F., 2019, Composición quími$\mathrm{ca}$, características físico-químicas, trazas metálicas y evaluación genotóxica del aceite de Plukenetia volubilis L. (sacha inchi). Revista Peruana de Medicina Integrativa, 4(1), 4. https://doi.org/10.26722/rpmi.2019.41.103

Arango, L., Idelka, Y., y Vega, D., 2020, Desarrollo Agroindustrial: Restricciones Económicas, Políticas y Naturales. Aglala, 11(S-1), 211-229. Recuperado de: http://revistas.curnvirtual.edu.co/index.php/aglala/article/view/1774

Arias Mesía, J., 2013. La Matriz BCG, su importancia, su estructura y desarrollo. Journal of Chemical Information and Modeling, 53(12) 1689-1699.

Ayaviri, V., Quispe, G., y Borja, M. E., 2017, El Capital Social en el desarrollo local comunitario. Un estudio en comunidades rurales de Bolivia. Revista Espacios, 38(43), 77-88. 
Bustamante, C., y Muñoz, Y., 2017, Propuesta de negocio inclusivo: hacia un modelo de inclusión social de sacha inchi en el Bajo Cauca antioqueño. Equidad y Desarrollo, 1(27), 125. https://doi.org/10.19052/ed.3834

Chiavenato, I., 2017, Comportamiento organizacional. La dinámica del éxito de las organizaciones. México: McGrawHill.

Consejo Municipal del Tambo, 2020, Plan de desarrollo municipal Tambo, Cauca 2020-2023.pdf (pp. 1-204).

David, F. R., 2013, Conceptos de Administración Estratégica (14a ed.). Pearson, México. https://issuu.com/victore.cardozodelgado/docs/ conceptos_de_administraci_n_estrat

Estrada, H. H., Saumett, H. G., y Bahamón, M. J., 2017, Direccionamiento estratégico y aprendizaje organizacional en unidades productivas asociadas a cooperativas del sector agrícola del Atlántico. Revista Espacios, 38(37), 19.

Fmcg \& Retail, 2018, 4 de cada 10 colombianos están cambiando a la versión saludable de su producto preferido. NielsenIQ. https://nielseniq.com/global/ es/insights/analysis/2018/4-de-cada-10-colombianos-estan-cambiando-a-la-version-saludable-de-su-producto-preferido/

Gómez, D., y Montaña, E., 2019, Caracterización en el eslabón de comercialización en la cadena de valor del cultivo de Sacha Inchi (Plukenetia volubilis) en el departamento del Meta. Trabajo de Fin de Grado, Universidad de Santo Tomás. https:// repository.usta.edu.co/handle/11634/18025

Hamel, G., y Prahalad, C. K., 2018, Do you really have a global strategy? En Buckley, P. J. (ed.), International Business (pp. 285-294). Routledge, Londres. https:// doi.org/10.4324/9781315199689-18

Hernández, R., 2018, Metodología de la investigación: las Rutas cuantitativa, cualitativa y mixta. McGraw Hill, México.

Jaya, P.E.J. y Yuliarmi, N.N., 2019, Factors influencing competitiveness of small and medium industry of Bali: Porter's five forces analysis. RJOAS: Russian Journal of Agricultural and Socio-Economic Sciences, 89(5). doi 10.18551/rjoas.2019-05.06 https://rjoas.com/issue-2019-05/article_06.pdf

Jodar Marco, C., 2018, Snackificación: un nuevo concepto de consumo. Ainia. Recuperado de: https:/www.ainia.es/tecnoalimentalia/consumidor/snacksnuevo-concepto-consumo/

López, K., Santa Cruz, C., y Gutiérrez, A., 2001, Perfil de Proteínas de las Semillas de "Sacha Inchi" (Plukenetia volubilis L. y Plukenetia huayllabambana Bussmann). The Biologist, 1(2), 11-20. https://doi.org/10.24039/rtb201614181

Ministerio del Interior y de Justicia, 2009, Municipio de El Tambo Comité Local para la Prevención y Atención de Desastres CLOPAD. Recuperado de: https:// repositorio.gestiondelriesgo.gov.co/bitstream/handle/20.500.11762/28532/ Caracterizacion_Tambocauca_2009.pdf?sequence $=2$ 2 2 isAllowed $=$.

Obando, E., 2013, Planeación por necesidades. Corregimiento de Quilcacé-Municipio del Tambo (Cauca). Tesis de maestría. Universidad Autónoma de Manizales. Recuperado de: http://repositorio.autonoma.edu.co/bitstream/11182/288/1/ 
Planeaci\%c3\%b3n_necesidades_corregimiento_Quilcac\%c3\%a9_municipio_ Tambo_Cauca.pdf.

Pitre, R., Herández, H., y Sierra, Á., 2020, Creación de empresas en Colombia: un análisis del perfil del emprendedor moderno. Clío América, 14(28), 2389-7848. https://doi.org/10.21676/23897848.3854

Rivera, C., y Labrador, O., 2013, Bases teóricas y metodológicas de la cooperación y el cooperativismo. Revista Cooperativismo y Desarrollo, 1(2), 191-208. https:// dialnet.unirioja.es/servlet/articulo?codigo $=5233964$

Rogel, A., y Yumaria, K., 2019, La cadena de valor para la comercialización con valor agregado del Sacha Inchi. Tesis profesional. Universidad Técnica de Machala. Recuperado de: http://repositorio.utmachala.edu.ec/handle/48000/14504

Sachacolombia, 2017, Nuestra historia. SumaSach'a. Recuperado de: https:// sumasacha.com/pages/nuestra-historia.

Schultz, T. W., 1967, Efficient Allocation of Brains in Modernizing World Agriculture. Journal of Farm Economics, 49(5), 1071-1082. https://doi.org/10.2307/1236985

Steenkamp, J., Cilliers, E. J., Cilliers, S. S., y Lategan, L., 2021, Food for thought: Addressing urban food security risks through urban agriculture. Sustainability, 13(3), 1-29. https://doi.org/10.3390/su13031267

Wang, S., Zhu, F., y Kakuda, Y., 2018, Sacha inchi (Plukenetia volubilis L.): nutritional composition, biological activity, and uses. Food Chemistry, 265, 316-328. https://doi.org/10.1016/j.foodchem.2018.05.055 